\title{
Phenomenological Study of Hysteresis in Quasistatic Friction
}

\author{
Lydéric Bocquet $\left({ }^{1,2, *}\right)$ and Henrik Jeldtoft Jensen $\left({ }^{2}\right)$

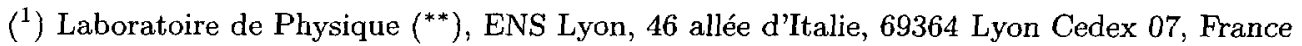 \\ $\left({ }^{2}\right)$ Department of Mathematics, Imperial College, 180 Queen's Gate, London SW7 2BZ, UK
}

(Received 27 February 1997, revised 21 May and 7 July 1997, accepted 1 September 1997)

PACS.46.30.Pa - Friction, wear, adherence, hardness, mechanical contacts, and tribology PACS.05.70.Ln - Nonequilibrium thermodynamics, irreversible processes

\begin{abstract}
Recently, hysteretic behaviour has been measured in some friction experiments involving elastrc surfaces. Moreover the approach toward the asymptotic value for the friction force is characterized by a well defined length scale. We describe how this behaviour is connected to the collective properties of the elastic instabilities suffered by the elastic body as it is displaced quasistatically. We express the relaxation length of the hysteresis in terms of the elasticity of the surface and the properties of the rough substrate. The predicted scaling are confirmed numerically.
\end{abstract}

Résumé. - Des résultats expérimentaux récents ont montré que la force de friction entre deux surfaces élastıques présente un comportement hystérétique. De plus, la relaxation de la force de friction vers sa valeur asymptotique est caractérisée par une longueur. Nous décrivons comment ce comportement est lié aux propriétés collectives des instabilités élastiques subies par le solide lorsqu'il est déplacé quasistatiquement. La longueur de relaxation est exprimée en terme de l'élasticité de la surface et des propriétés du support rugueux. Les lois d'échelle prédites sont confirmées numériquement.

\section{Introduction}

The present paper discusses the origin of hysteretic behaviour and history dependent effects measured in some recent friction experiments. We use a simple model to develop a phenomenological analysis of hysteresis in friction in the quasi-static limit (zero velocity, r.e., below the depinning threshold). In particular, we emphasize the connection between the measured hysteresis of the friction force and the microscopic elastic instabilities occurring when an elastic surface is pulled over a microscopically rough surface. Guided by computer simulations of a one-dimensional elastic chain, we construct a coherent collective pinning picture of friction. In particular, an equation for the relationship between the friction force and the number of elastic instabilities is derived. We use this equation to obtain the hysteretic relation between displacement and friction force. The relevance of our model to describe experimental results is discussed.

$\left(^{*}\right)$ Author for correspondence: (e-mail: lbocquet@physique.ens-lyon.fr)

$\left({ }^{* *}\right)$ URA CNRS 1325

(C) Les Éditions de Physique 1997 
1.1. Hysteresis in the Elastic Limit. - Friction between two solid bodies is found experimentally to obey the two amazingly simple Amonton's laws, namely that: i) the frictional force is independent of the size of the surfaces in contact; ii) friction is proportional to the normal load.

In spite of their simplicity, the understanding of these laws remained obscure for a (long) while. The first step was done by Coulomb, who pointed out that roughness was responsible of the interaction forces between the surfaces. The friction force then originates from the interlocking of a number of asperities on the two surfaces. But the first statisfactory understanding only came out in the first half of this century. On the base of the systematic experimental investigation of many friction phenomena, Bowden and Tabor then proposed the first "modern" theory of friction [1]. Their point is that the real contact area between the two solids is only a small part of the "bare" contact area, so that the asperities in contact undergo large pressures and thus deform plastically. Starting from these two ideas, they could justify the two Amonton's laws. However, since then, the development of clean, reproducible experiments indicated the limitations of these laws (see e.g. [2] and references therein) and contributed to refine the theoretical understanding of friction phenomena [3-6].

In this paper, we shall restrict our attention to a specific feature observed in recent experiments of friction conducted in the "elastic" regime. These experiments exhibit hysteresis of the friction force as a function of the displacement of the solid body, so that it takes a finite distance for the system to reach the stationary sliding regime. This means that the friction force does not depend only on velocity or position, but on the displacement performed before reaching the point at which the force is measured [7]. These history dependent effects are thus characterized by a length scale, which we shall denote as "recovery" or "memory" length. This phenomena has been reported in AFM friction experiments at the nanometer scale [8,9], in experiments using artificial elastic surfaces with millimetric asperities [7], as well as in measurements in the related situation of the hysteresis of the contact line at the nanometer scale [10].

The existence of "memory effects" in dry friction characterized by a length is known since the experimental work of Rabinowicz [11] and was accounted for by Ruina and others by introducing new empirical state variables $[12,13]$. The "memory length" (of the order of the micrometre) is commonly interpreted as the distance needed to break a contact and originates from the (slow) plastic deformations of the contacts. Therefore the existence of hysteresis even in the elastic regime as measured in the previous experiments cannot be handled by such approaches and new mechanisms have to be found to understand it. One approach was proposed recently by Tanguy and Roux [14], who have shown that even in the elastic regime, memory effects characterized by a length can be induced by long-range correlations in the roughness (for example for self-affine surfaces). However this was not the case for the experiments cited above and another origin has to be found. This is the object of this paper.

Inspired by the previous experiments, we shall consider in a first step a much simplified version of the problem. Our model consists in a one-dimensional chain of springs, moving quasistatically on a rigid disordered surface (see Sect. 2.1 for details). Though very simple, we shall show that the model already exhibits history dependent effects characterized by a length scale in the building up of the friction force. The generalization of our results to twodimensional systems is straightforward. As we shall show, the crucial point is that in low dimension ( 1 and 2), a typical correlation length in the system is smaller than the system size and friction is a collective process. As it stands, our approach does not however apply to 3-dimensional systems. This point will be discussed in the conclusion.

1.2. The Crucial Role of Elastic Instabilities. - As already pointed out by Tomlinson [15], friction arises in an elastic media due to the existence of a multiplicity of metastable states. 


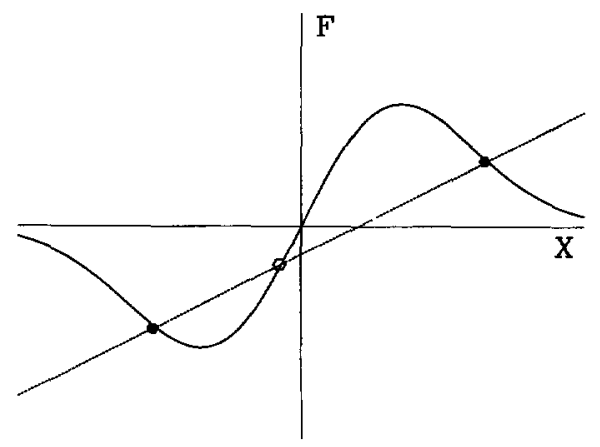

Fig. 1. - Graphic resolution of equation (2) (in the multistable case $2 A_{\mathrm{p}} / R_{\mathrm{p}}^{2}>\kappa$ ). The full line is the derivative of the defect Gaussian potential. The slope of the straight line is $\kappa$, the stiffness of the spring. The intersection gives the solution of equation (2). Black dots are the stable solutions, the open dot is the unstable solution.

This multiplicity induces mechanical instabilities in the system and leads to dissipation and hysteresis $[6,16,17]$.

The instabilities arise whenever the local force-balance equation describing the interlocked surfaces becomes multivalued. As the mutual displacement of the two bodies is increased, the asperities are forced against each other. The interface force increases linearly as a function of the centre of mass displacement. When the local force balance becomes unable to sustain the mutual force between the asperities, a local rearrangement of the atoms in the interface will take place. If the roughness of the interacting surfaces is sufficiently large, the motion of the interface atoms takes place in the form of swift jumps from one metastable configuration to another. During such an instability the friction force drops precipitously by a certain value, for then again to increase linearly upon further increase in the relative displacement of the two bodies.

Let us recall the microscopic features of the elastic instabilities. This is most easily done by use of a single degree of freedom picture $[6,17]$. Consider a particle at position $x$ elastically coupled to a position $X$ (one may think of $X$ as the center of mass of the elastic lattice). We want to follow the motion of the particle as it passes over an asperity modeled by a Gaussian peak in the potential energy. The energy of this system is given by

$$
U=\frac{1}{2} \kappa(x-X)^{2}+A_{\mathrm{p}} \exp \left[-\left(x / R_{\mathrm{p}}\right)^{2}\right],
$$

$\kappa$ being the stiffness of the elastic coupling, and $A_{\mathrm{p}}$ and $R_{\mathrm{p}}$ the strength and range of the asperity potential.

The static equilibrium of the system is obtained by solving the equation $\partial U / \partial x=0$ for a prescribed $X$ value, i.e., we have to solve the equation

$$
\kappa(x-X)=\frac{2 A_{\mathrm{p}} x}{R_{\mathrm{p}}^{2}} \exp \left[-\left(x / R_{\mathrm{p}}\right)^{2}\right] .
$$

This equation is solved graphically in Figure 1. The important point is that the equation has a single valued solution for any value of $X$ as long as the slope of the force exerted by the Gaussian peak is everywhere smaller than $\kappa$. This condition is

$$
\frac{2 A_{\mathrm{p}}}{R_{\mathrm{p}}^{2}}<\kappa \text {. }
$$


When this condition is not fulfilled (as in Fig. 1), the equilibrium position of the particle $x(X)$ will become a discontinuous function of $X$ : a mechanical instability occurs when the system passes over the defect. Accordingly, energy is dissipated (e.g. into the rapid degrees of freedom, like phonons): instabilities induce hysteretic behaviour. In a more general case where an elastic surface slides over many defects, one may still expect the basic mechanism for friction to be linked to the occurrence of elastic instabilities.

1.3. OUR AIMS. - Our aim in this paper is to give a mainly qualitative picture of collective pinning in the quasi-static limit in order to understand the memory effects. We stress the fact that we shall study the system in a regime where the sliding velocity of the two solid bodies strictly vanishes. In other words we study the system in a regime where the external force applied on the system is less than the critical value $F_{\mathrm{c}}$ above which the system acquires a finite, non vanishing velocity. This transition is usually denoted as the depinning transition, and $F_{\mathrm{c}}$ as the critical pinning force. Thus no dynamical effects are expected in our case and the system will be considered at equilibrium under an applied external force. The velocity dependence of the friction force is therefore not the purpose of the present study [18-20].

We will attack the problem along the following line:

i) First we perform numerical simulations of a very simple model of the system, in order to check the existence of hysteretic effects characterized by a length scale.

ii) We then construct a "microscopic" scenario of friction by analysing the microscopic behaviour of the system.

iii) Finally, on the basis of the numerical results, we propose a qualitative phenomenological model for the collective properties of friction in the quasi-static limit. This model provides the link between the collective equilibrium and out-of-equilibrium properties of the system below the depinning threshold.

\section{General Features of the Numerical Results}

2.1. A Simple Numerical Model. - Let us first introduce the model we use for our numerical experiments. For simplicity we model the two interacting elastic surfaces by a mobile deformable elastic medium in contact with a stationary undeformable rough surface. This is clearly a limitation in comparison to two deformable elastic surfaces. However, our findings will justify our anticipation that this simplification is inessential.

Our model consists of a one-dimensional string of particles at positions $x_{z}$ where $i=1, \ldots, N$, coupled together through elastic springs all of the same spring constant $k$. The chain, of length $L$, is assumed to be periodic. We use the equilibrium length $a$ of the elastic springs as our unit of length $a=1$. The particles interact with a set of pinning centres in the form of randomly positioned (at positions $x_{\imath}^{\mathrm{p}}$ where $i=1, \ldots, N_{\mathrm{p}}$ ) repulsive asperities of density $n_{\mathrm{p}}$. All the asperities are modelled by the same Gaussian potential peak of amplitude $A_{\mathrm{p}}$ and range $R_{\mathrm{p}}$. The potential energy of the system can accordingly be written as

$$
\begin{aligned}
U & =U_{\mathrm{el}}+U_{\mathrm{pin}} \\
& =\frac{k}{2} \sum_{\imath=1}^{L-1}\left(x_{\imath}-x_{\imath+1}-a\right)^{2}+\sum_{\imath=1}^{L} \sum_{\jmath=1}^{N_{\mathrm{p}}} A_{\mathrm{p}} \exp \left[-\left(x_{\imath}-x_{\jmath}^{p}\right)^{2} / R_{\mathrm{p}}^{2}\right]
\end{aligned}
$$

We are interested in experiments where dynamical effects can be neglected. For this reason we investigate the total force produced by the asperities when the elastic chain is moved quasistatically through the asperities. 
The quasistatic motion of the chain has been performed numerically using a Molecular Dynamics (MD) annealing method, developed previously for such systems [17]. We recall here the main points of the relaxation method. First, a unit mass is ascribed to each particle of the chain. Then, MD equations of motion are written, which are derived from the potential in equation (4) supplemented by an auxiliary kinetic term. Note that in our simulations, the averaged kinetic energy per particle, i.e. the temperature, is taken to be very low (of order $10^{-4} A_{\mathrm{p}}$ ). The numerical integration of the equations of motion is done using the leap-frog algorithm [17]. The time-step for the integration was taken to be $\delta t=0.01(\mathrm{~m} / \mathrm{k})^{1 / 2}$

Now, starting from a given configuration with c.o.m. position $X_{\text {old }}$, the elastic chain is first displaced as a rigid body by a small amount $\mathrm{d} x\left(\mathrm{~d} x / a=10^{-5}\right.$ in our simulations $)$ by replacing all the particle positions by $x_{\imath} \mapsto x_{\imath}+\mathrm{d} x$. The chain is then relaxed to the asperities using the MD procedure during 10 time steps. We checked that the results (in particular the friction force versus c.o.m. position) do not change upon further relaxation, i.e. by increasing the number of relaxation steps per infinitesimal shift. The c.o.m. is kept fixed by always counteracting the force exerted by the substrate by an external force $f_{\text {ext }}$ applied homogeneously to all the particles of the chain. We have

$$
f_{\mathrm{ext}}=\frac{1}{L} \sum_{\imath=1}^{L} \frac{\partial U_{\mathrm{pin}}}{\partial x_{\imath}}
$$

where $f_{\text {ext }}$ used in the present time step is calculated from the positions of the previous molecular dynamics time step. At the end of each of these relaxation procedures, the excess kinetic energy is extracted from the system by rescaling all the velocities by the square root of the ratio of the (measured) final over the (ascribed) initial kinetic energy of the system. Note that the small kinetic energy put in the system is only used as a numerical trick to let the system move and explore locally its phase space. This procedure of successive shift and relaxation is then iterated, until the c.o.m. has been shifted a distance of a few lattice spacings $a$. This method allows one to follow as accurately as desired the motion through the background potential and a whole curve of the friction force $F_{\mathrm{f}}$ versus c.o.m. position $X_{\text {c.o.m }}$ can be constructed. Simulations have been performed for two system sizes $(N=500$ and $N=1000)$, the results being similar within the statistical uncertainties (although longer runs were necessary for the longer chain).

Whether this MD annealing method produces the "correct" set of metastable configurations or not is a delicate question. In order to answer this problem, we compared our results with constant force simulations using overdamped dynamics, which is the "standard" way of simulating such systems [18]. Starting from a given configuration characterized by a c.o.m. position $X_{\text {old }}$ and a force $F_{\text {ext }}^{0}=F_{\mathrm{f}}\left(X_{\text {old }}\right)$, a small shift in the force, $\delta F$ is imposed. The system then move till the sum of the forces vanishes, leading to a new c.o.m. position $X_{\text {new }}$, so that $F_{\text {ext }}^{0}+\delta F=F_{\mathrm{f}}\left(X_{\text {new }}\right)$. Note that this is correct for forces under the depinning force $F_{c}$ (since the system will acquire a finite velocity for $F_{\text {ext }}>F_{c}$ ), but this is anyway the part of the problem we are interested in.

The important point is then that these constant force simulations give results in qualitative and quantitative agreement with those obtained using the MD annealing method, thus confirming the validity of this procedure to drive the system. The draw back of simply applying a constant external force is that the force from the substrate fluctuates in space. A constant force will therefore sometimes be much larger than the pinning force. This leads to unwanted acceleration effects and make it difficult to remain in the limit of quasistatic motion [21]. The results presented below were obtained using the MD annealing method. 


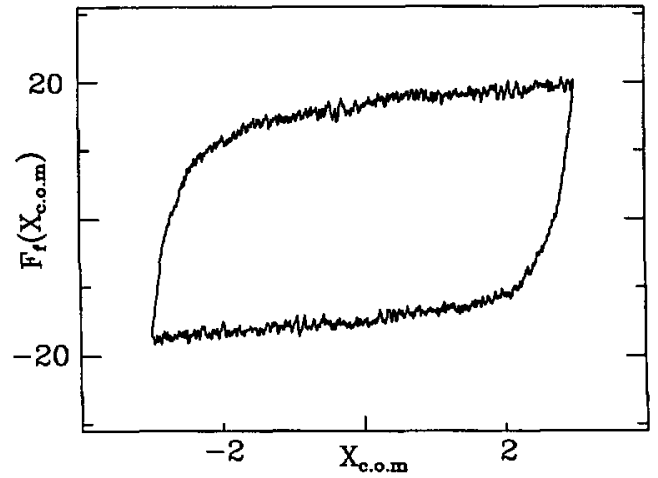

Fig. 2.

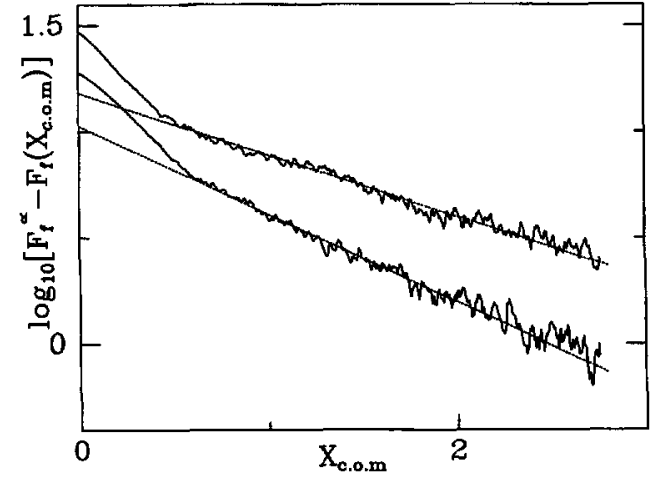

Fig. 3.

Fig. 2. - Hysteresis of the averaged force as a function of the center of mass displacement $X_{\mathrm{c} o \mathrm{~m}}$ when the elastic chain is pulled back and forth over the defect surface. The parameters are: length of the elastic chain $N=500$; density of pinning centres $n_{\mathrm{p}}=0.5$; parameters of the Gaussian defect potential $A_{\mathrm{p}}=0.06, R_{\mathrm{p}}=0.25$.

Fig. 3. - Log-linear plot of the averaged force as a function of the center of mass displacement $X_{\mathrm{c} \text { o } \mathrm{m}}$ for two different densities (top curve $n_{\mathrm{p}}=0.35$, bottom curve $n_{\mathrm{p}}=0.2$ ). The dotted lines are an exponential fit of the averaged force in the large $X_{\mathrm{c} \circ \mathrm{m}}$ limit. The other parameters are $N=500$, $A_{\mathrm{p}}=0.06$ and $R_{\mathrm{p}}=0.25$.

2.2. Existence of Hysteresis. - Inspired by the experiments of reference [7], we move the elastic chain back and forth over the "rough" surface, according to the numerical algorithm

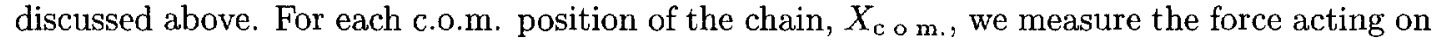
the particles of the elastic chain. This force will be denoted as the friction force, $F_{\mathrm{f}}$. The whole curve $F_{\mathrm{f}}$ versus $X_{\text {c.o.m. }}$ is then averaged over many cycles and over different random spatial configurations of pinning centres. Figure 2 shows a typical numerical result. Starting from a given point, the friction force reaches after a finite distance a plateau value, independent of the c.o.m. position. This plateau value is the static friction force: it is the maximum value for an external force before the solid body (here. the elastic chain) acquires a non vanishing velocity. Then, when the system is moved in the other direction, the same plateau value with the opposite sign is reached, but following a different curve in the $F_{\mathrm{f}}$ versus $X_{\mathrm{c} . \mathrm{o} \text { m. plane: an }}$ hysteresis loop is performed during a cycle.

As in the experiments, history dependent effects are clearly observed, since it takes a finite length for the system to reach the stationary pinning force. This defines a recovery length.

2.3. Exponential Decay of the Pinning Force. - As shown in Figure 3, the approach towards the plateau value for the friction force is exponential. For example, when the system is pulled in the $X_{\text {c.o.m }}>0$ direction, the friction force can be very well fitted after an initial small transient distance, by the following relation:

$$
F_{\mathrm{f}}\left(X_{\mathrm{co} . \mathrm{m}}\right)=F_{\infty}+\left(F_{0}-F_{\infty}\right) \exp \left\{-\frac{X_{\mathrm{c} . \mathrm{om}}-X_{0}}{\zeta}\right\}
$$

where $F_{\infty}$ is the plateau value for the force, $F_{0}$ is the force measured at a given point $X_{0}$. This relation defines the recovery length, $\zeta$. In our simulation, the latter was obtained to be 


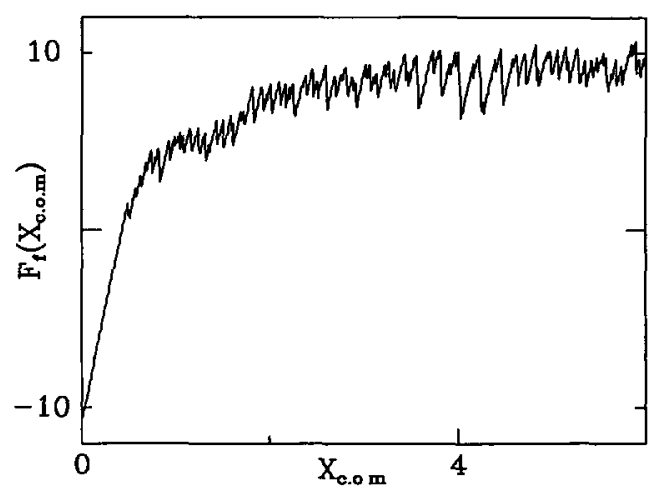

Fig. 4. - Plot of the non-averaged force as a function of the center of mass displacement, z.e., for one realization of the cycle. The parameters are $N=500, n_{\mathrm{p}}=0.2, A_{\mathrm{p}}=0.06$ and $R_{\mathrm{p}}=0.25$.

of the order of a few $R_{\mathrm{p}}$, the range of the defects. The size of the initial transient regime was obtained to decrease when the density of pinning centres $n_{\mathrm{p}}$ increases.

One can note that the same exponential approach towards the plateau value of the pinning force has been found experimentally in references $[7,10]$. This point will be discussed in the conclusion. We now analyse in greater details the simulation results to give a microscopic picture leading to these results.

\section{A First Empirical Understanding}

3.1. HoOkE's LAW And InstabiLities. - While in Figure 2, we considered an average of the friction force over many different initial states, we now focus our attention on a particular realization of the numerical experiment. A typical non-averaged plot of the friction force as a function of the c.o.m. displacement is shown in Figure 4. This figure is characterized by a saw-tooth behaviour, which splits up into linear increase of the friction force, separated by steep decrease in the friction force.

(i) The linear behaviour corresponds to the reversible linear (elastic) response of the system, when an external force is applied in order to impose a given c.o.m. displacement. This response is characterized by an elastic susceptibility, $\kappa_{\mathrm{L}}$, defined by

$$
\mathrm{d} F_{\mathrm{f}}=\kappa_{\mathrm{L}} \mathrm{d} X_{\mathrm{com}} .
$$

relating the measured infinitesimal change in the friction force, $\mathrm{d} F_{\mathrm{f}}$, to the c.o.m. displacement $\mathrm{d} X_{\text {c.o.m. }}$, according to a simple Hooke's law. The parameter $\kappa_{\mathrm{L}}$ was first introduced by Labusch [22], in the context of lattice deformations in crystals and will be denoted as the "Labusch parameter" in the following.

(ii) The discontinuities in the force are the indication of a dramatic irreversible transformation occurring in the system. A look at the microscopic trajectories shows that these jumps in the force are intimately connected to a large common displacement of a significant number of particles (typically of order 10-50 among 500). The typical displacement $\delta x_{0}$ of each particle of the moving block was always of the order of one lattice spacing in all our simulations: $\delta x_{0} \simeq a$. This indicates that. because of the imposed c.o.m. displacement, the local equilibrium state of the system becomes unstable and a finite jump occurs towards a new local equilibrium state [17]. In other words, these jumps occur because the system cannot support elastically 
the applied external force anymore, so that a new metastable state has to be found in order to release the stored energy. Dissipation occurs during these abrupt transitions. In the following, these jumps will be denoted as "elastic instabilities".

The previous scenario is in fact very reminiscent of the single particle case (i.e., one spring over one defect), discussed above $[6,23]$. In particular, the multistability of the metastable positions seems thus to be recovered in the many-particle system. However the characterization of the associated instabilities is now much more difficult, because of the collective character of the phenomena, as will be shown in Section 4 .

Let us assume that the elastic chain is pulled in the $X_{\mathrm{com}}>0$ direction. We introduce the spatial frequency of instabilities, $\nu$, defined as the number of instabilities occurring in the system per unit c.o.m. displacement; and $\Delta F_{\text {inst }}$ the drop in force occurring in a single instability. It is useful to define it to be the absolute value of the drop, so that $\Delta F_{\text {inst }}>0$. The latter is assumed, of course, to depend on the c.o.m. position (or equivalently on the friction force) at which the instability takes place. According to the previous scenario, a simple differential equation for the (averaged) friction force, $F_{\mathrm{f}}$, can be written:

$$
\text { - } \frac{\mathrm{d} F_{\mathrm{f}}}{\mathrm{d} X_{\text {c.o.m. }}}=\kappa_{\mathrm{L}}-\nu\left\langle\Delta F_{\text {inst }}\right\rangle
$$

which accounts for both the elastic response of the system and the finite change in the force during instabilities. The notation $\langle.$.$\rangle means a non-equilibrium average over many different$ distributions of pinning centres for a given c.o.m. displacement, or equivalently for a given friction force. We omit the brackets for the Labusch parameter $\kappa_{\mathrm{L}}$, since the latter is found in the simulations to be independent of the friction force $F_{\mathrm{f}}$. This means that the elastic susceptibility of the system remains constant, when the friction force increases, r.e., when new metastable states are explored. This rather astonishing fact will be discussed briefly in Section 5 .

We can see in Figure 4 that no instability occurs until the force has reached the value $F_{\mathrm{f}}=0$ and a rather long elastic relaxation takes place up to that point: then $\nu=0$ for $F_{\mathrm{f}}<0$. In fact, this is to be expected since in our numerical procedure (involving an alternative forward/backward displacement of the whole system like in the real experiments), the initial state is not an equilibrium state but the stationary state of the system when it is pulled in the opposite direction. Therefore, until some stress is effectively supported by the system (i.e., $F_{\mathrm{f}}>0$ ), the system relaxes elastically the stress and no instability occurs. For $F_{\mathrm{f}}>0$, the instability process is turned on. The frequency of instabilities is found numerically to reach a plateau value in a very short distance (much smaller than the lattice spacing, $a$ ), followed by a small decrease towards its stationary value. This small $F_{f}$ dependence will be omitted in the following and the frequency will be considered as roughly constant (for $F_{\mathrm{f}}>0$ ).

3.2. A Phenomenological Law and the Exponential Decay. - The averaged change in force during an instability, $\left\langle\Delta F_{\text {inst }}\right\rangle$ does not remain constant when the c.o.m. is displaced. This can be seen for example in Figure 4, where the non-averaged friction force is plotted versus the c.o.m. displacement. The change in force during an instability is seen to increase when the system is pulled quasi-statically, so that $\left\langle\Delta F_{\text {inst }}\right\rangle$ is expected to increase when the friction force $F_{\mathrm{f}}$ increases. We conjecture a simple linear relationship between these two quantities:

$$
\left\langle\Delta F_{\text {inst }}\right\rangle=\delta F_{0}+\alpha F_{\mathrm{f}}
$$

where $\delta F_{0}$ and $\alpha$ are two phenomenological parameters

This "phenomenological law" has been checked in the simulations by plotting the averaged change in an instability as a function of the friction force measured just before the instability 


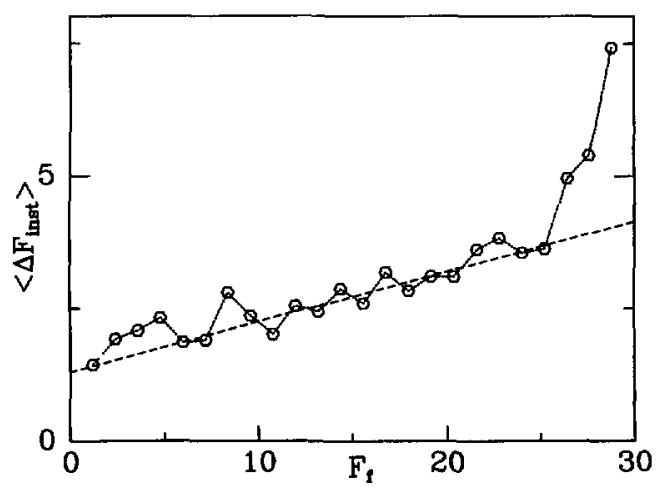

Fig. 5. - Average of the discontinuity in force during an instability, $\left\langle\Delta F_{\text {tnst }}\right\rangle$ as a function of the friction force $F_{\mathrm{f}}$. The dotted line is linear fit of the numerical points (the few last ones excepted). The slope gives the phenomenological parameter $\alpha$, while its value at the origin gives $\delta F_{0}$. For the numerical parameters under consideration $\left(N=500, n_{\mathrm{p}}=0.8, A_{\mathrm{p}}=0.06, R_{\mathrm{p}}=0.25\right)$, this gives $\alpha=0.095, \delta F_{0}=1.3$.

takes place. Numerically, this procedure involves a simple algorithm which detects the instabilities. The latter relies on the measure of the numerical derivative of the friction force, which exhibits a dramatic change during an instability. This rough indicator has been checked to work with a very good accuracy. We have then averaged the plot over many different realizations of the initial conditions to compute for a given friction force, the corresponding averaged change in force $\left\langle\Delta F_{\text {inst }}\right\rangle$. A typical result is plotted in Figure 5. Except in the large force region, the numerical points can be fitted with a good agreement by a straight line, thus validating the phenomenological relation in equation (9). Physically, the increase of the force release during an instability is understandable. Roughly, when the external force increases, the "susceptibility" of the system increases accordingly, so that the drop in force during an instability becomes larger. This point will however be studied in more details in the next section. The increase of $\left\langle\Delta F_{\text {inst }}\right\rangle$ for large forces in Figure 5 could be the indication of the onset of large fluctuations arising in the close vicinity of the depinning threshold, although "critical" avalanches ( $\iota . e$, involving the whole system) were not observed in our simulations. But this problem requires a specific careful numerical work as done by Pla and Nori [24]. This is not the object of the present work.

Combining the phenomenological relation (9) with equation (8) leads to a closed equation for the friction force:

$$
\begin{aligned}
\frac{\mathrm{d} F_{\mathrm{f}}}{\mathrm{d} X_{\text {c.o.m. }}} & =\kappa_{\mathrm{L}}-\nu\left\langle\Delta F_{\text {inst }}\right\rangle \\
& =\left(\kappa_{\mathrm{L}}-\nu \delta F_{0}\right)-\nu \alpha F_{\mathrm{f}} .
\end{aligned}
$$

This equation predicts an exponential relaxation of the friction force towards a stationary value given by

$$
F_{\mathrm{f}}^{\infty}=\frac{\kappa_{\mathrm{L}}}{\nu \alpha}-\frac{\delta F_{0}}{\alpha}
$$

The relaxation length, $\zeta$, is related to the phenomenological parameter $\alpha$, through the simple relation:

$$
\zeta=1 / \nu \alpha
$$


This exponential behaviour is in agreement with the numerical results (see Fig. 2).

Therefore, the phenomenological relation (9) provides an initial "empirical" understanding of the underlying physics of 1-D friction. However, how this simple law emerges from the microscopic picture remains to be clarified. Moreover, the results obtained within this approach for the stationary force (Eq. (11)) and relaxation length (Eq. (12)) are only useful, if some prediction can be made for their dependence on the physical parameters of the system (e.g. density of pinning centres $n_{\mathrm{p}}$, strength and range of the pinning centres, $A_{\mathrm{p}}, R_{\mathrm{p}}$ ). This is the object of the next section.

\section{Microscopic Picture: Towards Collective Pinning}

4.1. Description of Instabilities. - In Section 3.1 we described the instabilities as an instantaneous collective motion of an important number of particles of the system, while the other remained more or less stationary. Intuitively, it would be appealing to relate the drop in the friction force to the number of jumping particles during the instability. To this end, we have studied numerically the microscopic behaviour of the system when an instability takes place. Especially, after isolating an instability, we separated the system into the block of "jumping particles" and the remaining particles. For both parts of the system, we computed the change in the force due to the defects. This operation was repeated for a number of instabilities of different amplitudes, taking place at different c.o.m. positions. The general "rule" we obtained was the following: i) the change in force measured for the jumping particles only was found to be very small; ii) the global drop in the total friction force observed in an instability is caused by the remaining particles, which didn't undergo a large displacement. This can be interpreted as follows. When each of the $N_{\text {jump }}$ jumping particles move forward by a large, finite amount $\delta x_{0}$, the c.o.m. of the remaining particles will move backward by an amount $\Delta x_{\text {c.o.m. }} \simeq-N_{\text {jump }} \delta x_{0} / N$, in order to keep the c.o.m. of the complete system constant. The corresponding drop in force is then simply given by the relaxation of the corresponding elastic force, according to Hooke's law (see Eq. (7)):

$$
\begin{aligned}
F_{\text {after }}-F_{\text {before }} \equiv-\Delta F_{\text {lnst }} & =\kappa_{\mathrm{L}} \Delta x_{\mathrm{c} \circ \mathrm{m}} \\
& =-\frac{\kappa_{\mathrm{L}} \delta x_{0}}{N} N_{\text {jump }}
\end{aligned}
$$

Equation (13) provides the desired link between the drop in the force occurring in an instability and the corresponding "length" of the instability, i.e.. the number of particles that jumped, $N_{\text {jump. }}$

4.2. Existence of a Coherence Length. - The afore-mentioned point i) - that the change in force measured for the jumping particles only is extremely small - is very striking. In fact, this can be considered as a first indication of the collectrve character of the instabiluties. To understand this subtle point, we first recall how an instability occur in the single particle case, as discussed by Nozières and Caroli $[6,17]$. The system involves then only a single particle attached to a spring and interacting with a defect. The extremity of the spring is moved adiabatically. For a sufficiently soft spring (see Sect. 1.2 for details), the equilibrium position of the particle becomes multi-valued over a given range of system positions, i.e., the system becomes unstable. The evolution of the particle thus exhibits three phases when it encounters the defect: first an adiabatic move, corresponding to the elastic response of the defect; then a large finite jump towards a point where the reaction force is very small: this corresponds to the elastic "instability"; finally, an adiabatic move again, where only the tail 
of the defect's potential is felt by the particle. Accordingly, the drop in the force $\Delta f$ during the instability is of the order of the threshold force before the jump. Now consider $N_{\text {jump }}$ particles jumping according to such an "individual scenario", the global change in force for the whole system would simply be $\Delta F_{\text {inst }} \simeq N_{\text {Jump }} \Delta f$, which is proportional to the number of particles involved in the instability. This assumes that the drop in the force originates from the particles involved in the instability. But this differs dramatically from our observations in the numerical experiments. Indeed according to the previous points (i) and (ii), the crucial difference is that in our simulated systems, the drop in the force during an instability is only induced by the release of the elastic response of the particles not involved in the instability.

We interpret this point by assuming that the system separates into two types of particles: a few particles interact "strongly" with the defects, while all the other only interact weakly with the defects, i.e., they can be considered as "strongly correlated". The equilibrium positions of one (or a few) strongly pinned particle may become unstable, the latter thus performing a large finite jump. All particles "strongly connected" to this particle then simply follow collectively the jump of the first jumping particle. Within this scenario, the change in force due to the "jumping part" of the system has no reason to be large. Indeed, most of the jumping particles ( z.e., except for the one - or the few - strongly "pinned") do not interact strongly with the defects, so that their individual change in force during their jump is mostly random, justifying the observation facts (i) and (ii).

There is another indication of the "collective" character of the instabilities. Let us come back to the phenomenological relation (9) for the drop in the force during an instability, verified numerically in Figure 5. As can be observed in this figure, the value at the origin, $\delta F_{0}=\Delta F_{\text {inst }}\left(F_{\mathrm{f}}=0\right)$, is non-vanishing. This means that, as soon as instabilities are allowed, a finite (non-zero) number of particles are involved in the instability. Therefore, the length scale of the previously discussed "strongly connected" particles is non vanishing at equilibrium, and is hence an intrinsic property of the system at equilibrium.

These observations lead us to the notion of "strongly pinned" particles and "strongly connected" particles, the latter being characterized by a well defined correlation length. In fact, this separation is reminiscent of the analysis introduced by Larkin and Ovchinnikov (LO) $[25,26]$, of the pinning of vortex lines in superconductors. Their analysis is based on the introduction of a "correlation length", characterizing the balance between the elastic energy (of the Abrikosov lattice in superconductors) and the pinning energy due to the interaction with the defects. This length can be equivalently interpreted as the displacement correlation length of the system. More precisely, the linear size of the correlated volume $l_{\mathrm{L}}$ is defined as the length scale over which the elastic rigidity of the elastic medium is sufficient to counteract the random forces. The relative displacement induced by the random forces is small within a correlated length. The particles within a correlated length are strongly correlated in the sense that if a particle is displaced a small amount, this displacement will be transmitted by the rigidity of the lattice out to distances of the order of the size of the correlated volume. At distances larger than the size of the correlated volume the random forces become essential. The distortion of the elastic lattice becomes appreciable and particles farther apart than $l_{\mathrm{L}}$ are only weakly correlated. In this sense we can think of the elastic lattice as being broken into weakly interacting rigid lengths. It is at the interface between the correlated volumes that the elastic instabilities occur.

We now recall the (qualitative) LO argument in order to estimate $\ell_{L}$.

In the picture depicted above, the total pinning force on a correlated length, i.e., the force due to the defects, is the sum of many contributions of the same order $f_{0} \sim A_{\mathrm{p}} / R_{\mathrm{p}}$, but with different signs (since the strongly correlated particles only interact weakly with the pinning centres). Therefore, the pinning force on a correlated length involves a statistical summation 
over all the individual contributions and will be of order $N_{\mathrm{c}}^{1 / 2} f_{0}$, where $N_{\mathrm{c}}=n_{\mathrm{p}} \ell_{\mathrm{L}}$ is the number of pinning centres included in the correlated length (we now restrict our study to a 1 -D problem). Since there are $L / \ell_{\mathrm{L}}$ correlated segments in the system, the total pinning force is found to be of order:

$$
F_{\text {pin }} \sim L\left(\frac{n_{\mathrm{p}}}{\ell_{\mathrm{L}}}\right)^{1 / 2} f_{0} .
$$

Since this force only acts for a distance of order $R_{\mathrm{p}}$ (the range of the pinning potential), before changing randomly, the pinning energy is assumed to behave like

$$
\delta \mathcal{E}_{\mathrm{pin}} \sim L\left(\frac{n_{\mathrm{p}}}{\ell_{\mathrm{L}}}\right)^{1 / 2} f_{0} R_{\mathrm{p}} .
$$

On the other hand, the increase of elastic energy due to the deformation of the lattice inside a correlated length can be estimated as $\delta \mathcal{E}_{\mathrm{el}}=\frac{1}{2}\left(k / n \ell_{\mathrm{L}}\right) \delta x^{2}$, where $k$ is the bare spring constant, $n=1 / a$ is the density of particles ( $a$ is the spring length), and $\delta x$ is the distortion distance. The term $k / n \ell_{\mathrm{L}}$ takes into account the effectrve stiffness of $n \ell_{\mathrm{L}}$ springs contained in a correlated length. The distortion length is expected to be of order of $R_{\mathrm{p}}$, the range of the defect potential. For the whole system (involving $L / \ell_{\mathrm{L}}$ correlated volumes), we thus find

$$
\delta \mathcal{E}_{\mathrm{el}} \sim L \frac{1}{2} k a\left(\frac{R_{\mathrm{p}}}{\ell_{\mathrm{L}}}\right)^{2}
$$

Adding equations $(15,16)$, we find the change in energy per unit length associated with the randomly distributed defects:

$$
\delta \mathcal{F}=\frac{1}{2} k a\left(\frac{R_{\mathrm{p}}}{\ell_{\mathrm{L}}}\right)^{2}-\left(\frac{n_{\mathrm{p}}}{\ell_{\mathrm{L}}}\right)^{1 / 2} f_{0} R_{\mathrm{p}}
$$

The optimized correlation length is found by assuming that the system evolves towards a state which minimizes the costs in energy. This is done by minimizing the expression (17) with respect to $\ell_{\mathrm{L}}$, leading to

$$
\ell_{\mathrm{L}}=\left[\frac{2 k a R_{\mathrm{p}}}{n_{\mathrm{p}}^{1 / 2} f_{0}}\right]^{2 / 3}
$$

Replacing (18) into equation (14), we obtain a total pinning force which scales like

$$
F_{\mathrm{pin}} \sim L \frac{n_{\mathrm{p}}^{2 / 3} f_{0}^{4 / 3}}{\left(k a R_{\mathrm{p}}\right)^{1 / 3}} .
$$

Larkin and Ovchinnikov [26] assumed that the pinning force in equation (19) is the outof-equilibrium pinning force, defined as the force measured at the point where the systems depins, i.e., acquires a non-vanishing velocity. The latter corresponds to the asymptotic force measured in our numerical experiments when the system is pulled quasi-statically.

But our interpretation of $F_{\text {pin }}$ differs qualitatively from the previous affirmation. Indeed, the LO argument as depicted here is an equilibrium argument, which allows one to find the equilibrium properties of a lattice interacting with randomly distributed defects. In this case, the averaged pinning force should vanish (otherwise the system is not at equilibrium). Therefore the "pinning force" defined in equation (14) cannot be identified with the out-ofequilibrium pinning force. Moreover, the LO argument does not take the elastic instabilities 
into account, while the numerical simulations (and even the real experiments, see $[27,28]$ ) show that these instabilities play a crucial role in the pinning process.

In contrast to Larkin and Ovchinnikov, we interpret $F_{\text {pin }}$ of equation (14), obtained along the previous argument, as a typical underlying scale for the fluctuations of the reaction force due to the defects at equilibrium: more precisely, it fixes the amplitude of the root-mean-square (r.m.s.) fluctuations of the pinning force. How this scale is related to the non-vanishing, nonequilibrium pinning force (i.e., the plateau value for the force for example in Figure 2), remains to be understood and will be the object of the next sections.

4.3. Linking the Coherence Length and the Phenomenological Relation. - In order to make the connection between the equilibrium properties of the system and our empirical understanding of friction depicted in Section 3, we make the following two conjectures:

Assumption (A): in an instability, the number of particles undergoing a jump is given by the length of a characteristic jump-length. In the limit of zero applied force, this jump-volume is given by the Larkin length.

This assumption is intuitively understandable. Indeed, according to the picture already discussed in the previous paragraph, instabilities are initiated by the few particles which are strongly interacting with the defects. When one of these strongly interacting particles becomes unstable, and thus performs a large jump, all the particles which are strongly "attached" to this jumping particle will follow as a whole.

Assumption (B): when an external force is applied, the length of the jump length increases.

To lowest order in the external force, we may guess a linear increase of the correlation length (here identified as the jump length), as would be obtained by applying a kind of linear response theory to the system:

$$
\ell_{\mathrm{L}}\left(F_{\text {ext }}\right)=\ell_{\mathrm{L}}^{\mathrm{equ}}+\gamma F_{\text {ext }}
$$

where $\ell_{\mathrm{L}}^{\text {equ }}$ is the equilibrium correlation length obtained along the LO argument, defined in (18). $F_{\text {ext }}$ is the total external force applied to the system in the direction of the motion: $F_{\text {ext }}=\mathbf{F}_{\text {ext }} \cdot \hat{\mathbf{e}}$, with $\hat{\mathbf{e}}$ the unit vector pointing in the direction of motion. This guess is mainly empirical but will be confirmed a posteriori by the consistency of this assumption with the numerical results. However, it can be justified at least qualitatively by the following argument.

Let $L_{\mathrm{J}}$ denote the length of the jump-length and $n_{\mathrm{sp}}$ denote the density of strongly pinned particles. According to our picture, we have (in a one-dimensional system)

$$
L_{\mathrm{j}}=1 / n_{\mathrm{sp}}
$$

The density of strongly pinned particles will be related to the probability density $P_{0}(e)$ of elastic energy per particle. Indeed, according to the LO picture, the elastic chain keeps mainly its lattice spacing inside a correlated volume, while it is strongly distorted at the defects, i.e., for the strongly pinned particles. Therefore, the strongly pinned particles should be characterized by a large elastic energy, say greater than a given threshold value, $e_{\mathrm{th}} \sim k R_{\mathrm{p}}^{2}$. Hence,

$$
n_{\mathrm{sp}}=\frac{N}{L} \int_{e_{\mathrm{th}}}^{\infty} \mathrm{de} P_{0}(e)
$$

Now we apply an external force $f_{\text {ext }}$ per particle. Some of the initially strongly pinned particles will be able to relax their elastic energy, by "climbing up the pinning hill". Roughly, we can identify these particles with those whose elastic energy before the force was applied was less than $e_{\mathrm{th}}+\left|f_{\text {ext }}\right| R_{\mathrm{p}}$. We then deduce the change in the density of strongly pinned particles 
according to

$$
\begin{aligned}
\Delta n_{\mathrm{sp}} & =n_{\mathrm{sp}}\left(f_{\mathrm{ext}}\right)-n_{\mathrm{sp}}\left(f_{\mathrm{ext}}=0\right) \\
& =-\int_{e_{\mathrm{th}}}^{e_{\mathrm{th}}+\left|f_{\mathrm{ext}}\right| R_{\mathrm{p}}} \mathrm{de} P_{0}(e) .
\end{aligned}
$$

In other words, particles have to sustain stronger elastic energy in order to remain among the strongly pinned particles as the external force is applied.

We expand equation (23) for small $F$

$$
n_{\mathrm{sp}} \simeq n_{\mathrm{sp}}(F=0)-P_{0}\left(\mathrm{e}_{\mathrm{th}}\right)\left|f_{\mathrm{ext}}\right| R_{\mathrm{p}}
$$

The probability density $P_{0}\left(e_{\mathrm{th}}\right)$ is calculated at $f_{\text {ext }}=0$. It has dimension of an inverse energy and can accordingly be assumed to scale as the inverse of the LO (elastic or pinning) energy scale, i.e.,

$$
P_{0}\left(e_{\mathrm{th}}\right) \sim \frac{1}{e_{\mathrm{LO}}} \sim \frac{1}{F_{\mathrm{pin}} R_{\mathrm{p}} / L}
$$

where equations $(14,16,17)$ were used.

From equation (21) we arrive at the following expression for the jump-volume

$$
\begin{aligned}
L_{\mathrm{j}}\left(F_{\text {ext }}\right) & =L_{\mathrm{j}}^{\text {equ }}+\gamma\left|F_{\text {ext }}\right| \\
& =\ell_{\mathbf{L}}+\gamma\left|F_{\text {ext }}\right|
\end{aligned}
$$

where $F_{\text {ext }}=N f_{\text {ext }}$ is the total external force acting on the system. In the last equality we made use of the assumption that the jump-length approaches the Larkin length when the applied force approaches zero. The coefficient $\gamma$ is according to the above discussion given by

$$
\gamma \sim \ell_{\mathrm{L}} / F_{\text {pin }}
$$

where $l_{\mathrm{L}}$ and $F_{\text {pin }}$ are given in equations $(18,19)$ respectively.

By use of the two conjectures, we can now account for the "phenomenological relation", equation (9), relating linearly the drop in force measured an instability to the friction force. In our case, the external force applied in order to impose a given c.o.m. position is equal to the measured friction force, $F_{\mathrm{f}}$, so that $F_{\mathrm{f}}=F_{\text {ext }}$. Combining equations $(20,13)$, and setting $N_{\text {jump }}=n L_{\mathrm{J}}\left(F_{\mathrm{f}}\right)$ according to the previous argument, we obtain

$$
\begin{aligned}
\Delta F_{\mathrm{inst}} & =\frac{\kappa_{\mathrm{L}} n L_{\mathrm{J}}\left(F_{\mathrm{f}}\right) \delta x_{0}}{N} \\
& =\frac{\kappa_{\mathrm{L}} n \ell_{\mathrm{L}} \delta x_{0}}{N}+\left(\frac{\kappa_{\mathrm{L}} n \gamma \delta x_{0}}{N}\right) F_{\mathrm{f}} .
\end{aligned}
$$

The proposed scenario thus gives a coherent picture of the mechanism leading to the phenomenological law. Moreover, identifying this expression with the phenomenological relation equation (9), we are left with the following "microscopic" expressions for the two phenomenological parameters $\delta F_{0}$ and $\alpha$ :

$$
\begin{aligned}
\delta F_{0} & =\frac{\kappa_{\mathrm{L}} n \ell_{\mathrm{L}}^{\mathrm{equ}} \delta x_{0}}{N} \\
\alpha & =\frac{\kappa_{\mathrm{L}} n \gamma \delta x_{0}}{N} .
\end{aligned}
$$


One of the important consequences of equations (29) is that it will allow us to compute the dependence of the two introduced phenomenological quantities on the microscopic parameters of the system (density of pinning centres $n_{\mathrm{p}}$, strength and range of the defects $A_{\mathrm{p}}, R_{\mathrm{p}}$ ). This requires more information on the Labusch parameter $\kappa_{\mathrm{L}}$, which will be obtained in the next section.

For the dimensionless parameter $\alpha$, we obtain by use of equations $(27,29)$, the expression

$$
\alpha \sim \frac{\kappa_{\mathrm{L}}}{N} n \delta x_{0} \frac{\ell_{\mathrm{L}}^{3 / 2}}{L f_{0} n_{\mathrm{p}}^{1 / 2}}
$$

where the expression for $\ell_{\mathrm{L}}$ is given in equation (18). This expression can be written in a more transparent form:

$$
\alpha \sim \frac{\delta F_{0}}{F_{\mathrm{pin}}} .
$$

The predictions of equations (29.30) will be compared with the numerical results in Section 5.3. This will allow us to assess the validity of our approach. But we first need to characterize more properly the dependence of the other quantities, in particular of the Labusch parameter. This is done now.

\section{Friction and Scaling Laws}

5.1. The Labusch Parameter. - The Labusch parameter is defined in equation (7) as the elastic susceptibility of the system. By definition, this susceptibility measures the response of the pinning centres (defects) when the particles interacting through the springs are slightly displaced from their equilibrium positions, i.e., when the applied force balances the pinning force [21]. We performed numerically this "experiment" by displacing the c.o.m. by a very small amount and analysed the corresponding change in force on each defect. Surprisingly, the change in the reaction force of the defects was found to be homogeneously distributed over the defects, thus leading to a picture of an individual elastic response of the pinning centres to the displacement of the spring system.

Therefore, we expect the Labusch parameter to scale like

$$
\kappa_{\mathbf{L}}=n_{\mathrm{p}} L k_{0}
$$

where $n_{\mathrm{p}}$ is the density of pinning centres and $L$ the length of the system. $k_{0}$ is the individual elastic response of a defect, and can be roughly estimated as the probability for a defect to interact with a particle, of order $\sim n R_{\mathrm{p}}$ ( $n$ being the particle density and $R_{\mathrm{p}}$ the range of the potential), multiplied by a typical value of the second derivative of the pinning potential, of order $A_{\mathrm{p}} / R_{\mathrm{p}}^{2}$. This leads to

$$
k_{0} \sim n R_{\mathrm{p}} \frac{A_{\mathrm{p}}}{R_{\mathrm{p}}^{2}} .
$$

Combining, equations $(32,33)$, we obtain that the Labusch parameter should scale like

$$
\kappa_{\mathrm{L}} \sim n_{\mathrm{p}} A_{\mathrm{p}} R_{\mathrm{p}}^{-1}
$$

This scaling relation has been checked in the simulations, by varying $n_{\mathrm{p}}$ for a given set of potential parameters $A_{\mathrm{p}}, R_{\mathrm{p}}$, and varying $A_{\mathrm{p}}, R_{\mathrm{p}}$ for a given density. The numerical results are obtained to be in reasonable agreement with these predictions. The dependence on $n_{\mathrm{p}}$ is shown in Figure 8: the numerical value of $\kappa_{\mathrm{L}}$ is plotted as triangles; the underlying dotted 


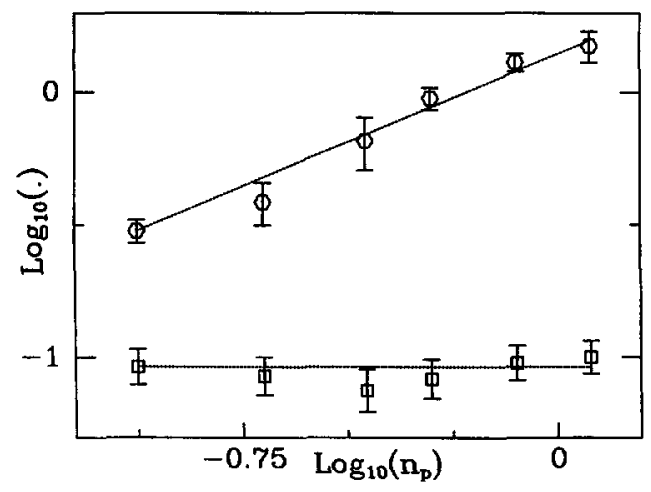

Fig. 6 .

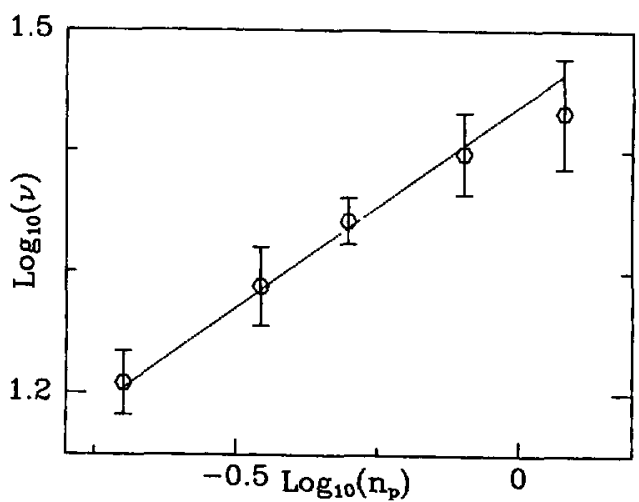

Fig. 7.

Fig. 6. - Log-Log plot of the two phenomenological parameters $\alpha$ (squares) and $\delta F_{0}$ (circles), as a function of the density of pinning centres $n_{\mathrm{p}}$. The straight lines show the predicted scalings: $n_{\mathrm{p}}^{0}$ for $\alpha$ and $n_{\mathrm{p}}^{2 / 3}$ for $\delta F_{0}$. The length of the chain is $N=500$ and the parameters for the potential of pinning centres are $A_{\mathrm{p}}=0.06, R_{\mathrm{p}}=0.25$.

Fig. 7. - Log-Log plot of the frequency of instabilities as a function of the density of pinning centres $n_{\mathrm{p}}$. The straight line has a slope $1 / 3$. The length of the chain is $N=500$ and the parameters for the potential of pinning centres are $A_{\mathrm{p}}=0.06 . R_{\mathrm{p}}=0.25$.

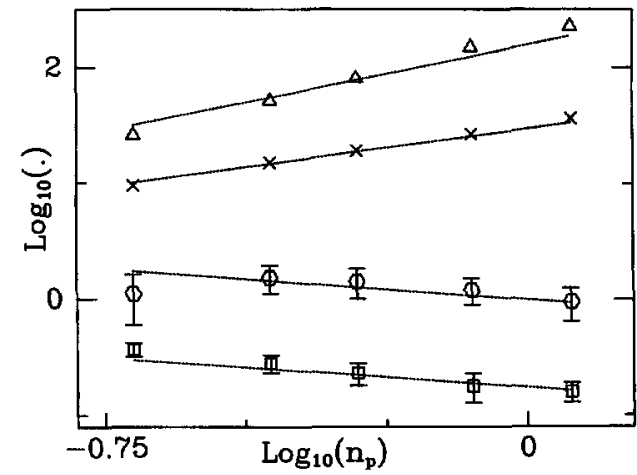

Fig. 8.

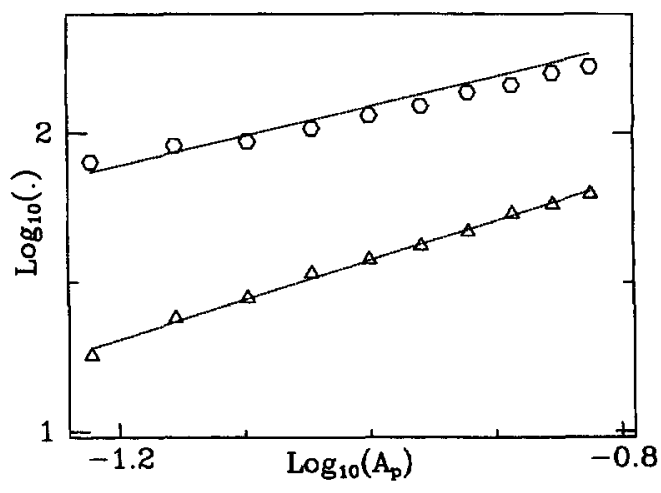

Fig. 9.

Fig. 8. - Log-Log plot of the different measured quantities as a function of the density of pinning centres $n_{\mathrm{p}}$. The triangles stand for the Labsuch parameter $\kappa_{\mathrm{L}}$; the crosses for the stationary static pinning force $F_{\mathrm{f}}^{\infty}$; the circles for the recovery length $\zeta$; and the squares for the length $\delta$. The straight lines indicate the scalings predicted within the proposed scenario. From bottom to top, the slopes of the lines are $-1 / 3,-1 / 3,2 / 3,1$. The length of the chain is $N=500$ and the parameters of the potential of pinning centres are $A_{\mathrm{p}}=0.06, R_{\mathrm{p}}=0.25$.

Fig. 9. - Log-Log plot of the stationary pinning force (triangles) and of the Labusch parameter (circles) as a function of the strength of the pinning centres, $A_{\mathrm{p}}$. The straight lines indicate the predicted scalings: $F_{\mathrm{f}}^{\infty} \sim A_{\mathrm{p}}^{4 / 3} ; \kappa_{\mathrm{L}} \sim A_{\mathrm{p}}^{1}$. The length of the chain is $N=500$ and the density of pinning centres $n_{\mathrm{p}}=0.5$. 


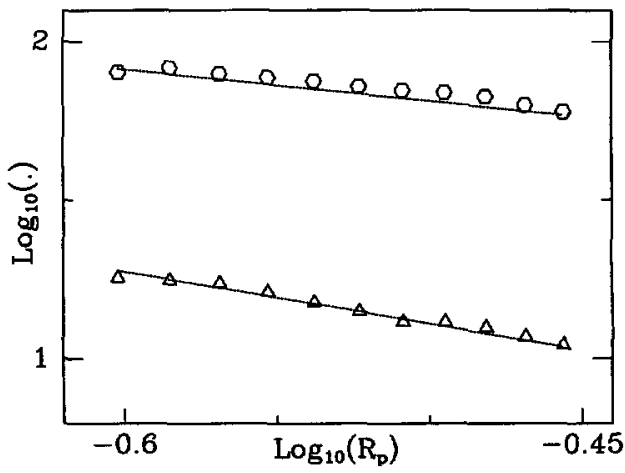

Fig. 10. - Same as Figure 9 but for the dependence on the range of the potential, $R_{\mathrm{p}}$. The straight lines indicate the predicted scalings: $F_{\mathrm{f}}^{\infty} \sim R_{\mathrm{p}}^{-5 / 3} ; \kappa_{\mathrm{L}} \sim R_{\mathrm{p}}^{-1}$. The length of the chain is $N=500$ and the density of pinning centres $n_{\mathrm{p}}=0.5$.

curve is a straight line with slope 1 . We explored numerically the dependence on the strength and range of the defect potential too. This was done by varying slowly $A_{\mathrm{p}}$ and $R_{\mathrm{p}}$ for a given density. The presented results do not involve however an average over different random spatial distributions of the pinning centres. The measured points for $\kappa_{\mathrm{L}}$ are plotted as circles in Figure 9 for the dependence on $A_{\mathrm{p}}$ and in Figure 10 for the dependence on $R_{\mathrm{p}}$ : The dotted lines are a guide for the eye to indicate the predicted slopes: slope 1 for the dependence on $A_{\mathrm{p}}$ (in Fig. 9) and slope -1 for the dependence on $R_{\mathrm{p}}$ (in Fig. 10). The trend is seen to be correct for both dependences. However a more extensive numerical study is still needed for a whole set of parameters $A_{\mathrm{p}}$ and $R_{\mathrm{p}}$, using different densities of pinning centres.

It is interesting to note that the picture of an individual response of the pinning centres for the elastic susceptibility of the system is coherent with the fact that the Labusch parameter is found in the simulations to be independent of the friction force (i.e., of the applied force). We confirmed this result in all our simulations, by checking that the slope of the linear part of the non-average friction force, as in Figure 4, does not change when the c.o.m. of the system is displaced. Apart from the previous qualitative argument, based on an individual response of the pinning centres, this result remains up to now quite obscure to us.

5.2. The Frequency of Instabilities. - Finally, to complete our phenomenological description, we need to characterize more precisely the frequency of instabilities, $\nu$. Up to now we do not have a full understanding of the mechanism of creation of instabilities, but some predictions can however be made.

In the stationary regime, the following relation is obtained from equations $(8,28)$

$$
\kappa_{\mathrm{L}}=\nu \Delta F_{\mathrm{inst}}=\nu \frac{\kappa_{\mathrm{L}} n \ell_{\mathrm{L}}\left(F_{\mathrm{f}}^{\infty}\right) \delta x_{0}}{N}
$$

where $F_{\mathrm{f}}^{\infty}$ is the plateau value of the friction force. This equality imposes the scaling of the asymptotic frequency $\nu$ to be

$$
\nu \sim \frac{N}{n \delta x_{0}} \frac{1}{\ell_{\mathrm{L}}^{\mathrm{equ}}} .
$$

Note that here we are only interested in the scaling properties of the frequency $\nu$, so that we forget any friction force dependence of $\ell_{\mathrm{L}}$ to focus on its dependence on density of defects 
and other microscopic parameters: we simply use the fact that the stationary value of the correlation length $\ell_{\mathrm{L}}\left(F_{\mathrm{f}}^{\infty}\right)$ has the same scaling on $n_{\mathrm{p}}$, etc... as $\ell_{\mathrm{L}}^{\text {equ }}$ defined in equation (18). We cannot a prior apply the expression for $\nu$ in equation (36) to the transient regime.

However an alternative argument can be given, which estimates $\nu$ from the fact that the system is broken up into the weakly and strongly pinned particles. Remember the mechanism producing the instabilities in the single particle problem (see 1.2): a particle $\mathrm{P}$ (position $x$ ) is elastically coupled to a position $X$ and interact with a defect. In the elastic region, we may approximate the force due to the defect by a Hooke's law, with a stiffness $k_{\mathrm{d}} \sim A_{\mathrm{p}} / R_{\mathrm{p}}^{2}$. Then, if the position $X$ is displaced by a small amount $\delta X$, the equilibrium position of the particle $\mathrm{P}$ will be displaced by an distance $\delta x \simeq \kappa \delta X /\left(\kappa+k_{\mathrm{d}}\right)$, where $\kappa$ is the spring constant. If the stiffness of the spring is much weaker than $k_{\mathrm{d}}$ (which is a condition for the existence of instabilities, see e.g. (3)), we obtain $\delta x \sim \kappa \delta X / k_{\mathrm{d}}$. Now in our many body system, we can

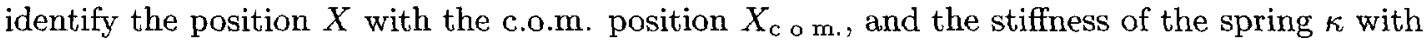
the effective stiffness of the correlated volume, $\kappa \sim k /\left(n \ell_{\mathrm{L}}\right)$. Moreover the particle $\mathrm{P}$ can be identified with one strongly pinned particle, since in our microscopic scenario the latter are expected to induce the instabilities. An instability will occur when the latter will move over a distance of order of the range of the potential: $\delta x \sim R_{\mathrm{p}}$. This is equivalent to a change in c.o.m. position $\delta X$ given by $\delta X \sim n \ell_{\mathrm{L}} k_{\mathrm{d}} R_{\mathrm{p}} / k$, according to our single particle discussion. This argument thus predicts a frequency scaling like

$$
\nu \sim \frac{k}{k_{\mathrm{d}} n R_{\mathrm{p}}} \frac{1}{\ell_{\mathrm{L}}^{\mathrm{equ}}}
$$

which is consistent - though not strictly identical - with our first guess, equation (36).

In particular, this argument predicts a dependence of the frequency of instabilities on the density of pinning centres as $\nu \sim n_{\mathrm{p}}^{1 / 3}$ (see Eq. (18)). The numerical results are consistent with this scaling as shown in Figure 7: the circles are the numerical points; the dotted line has a slope $1 / 3$.

5.3. Summary of THE PRedictions. - At this stage, we can check the predictions for the phenomenological parameters and measured quantities obtained within our scenario: in particular for the two phenomenological parameters $\delta F_{0}$ and $\alpha$, for the Labsuch parameter, the plateau value of the friction force $F_{\mathrm{f}}^{\infty}$ and the recovery length $\zeta$. Apart from the pinning force and the Labusch parameter, we have mainly focussed our numerical study on the dependence of the measured quantities on the density of pinning centres $n_{\mathrm{p}}$. Since the predicted scalings on $n_{\mathrm{p}}$ are non trivial (see the different powers of $n_{\mathrm{p}}$ in the equations below!), the comparison with the numerical results as a function of $n_{\mathrm{p}}$ are therefore expected to be already a drastic test for our scenario. But clearly more extensive numerical work needs to be done to explore the whole parameter space $\left(n_{\mathrm{p}}, A_{\mathrm{p}}, R_{\mathrm{p}}\right)$.

First we focus on the phenomenological parameters, $\delta F_{0}$ and $\alpha$. Their "microscopic" expressions are given in equations $(29,30)$. Combining the predicted scaling of the Labusch parameter, as displayed in equation (32) with the expression of the correlation length $\ell_{\mathrm{L}}^{\text {equ }}$, given in equation (18), we obtain the following dependence as a function of the density of pinning centres, $n_{\mathrm{p}}$ :

$$
\begin{aligned}
\delta F_{0} & \sim n_{\mathrm{p}}^{2 / 3} \\
\alpha & \sim n_{\mathrm{p}}^{0} .
\end{aligned}
$$

In particular, this shows that the slope $\alpha$ of the phenomenological law equation (9) does not depend on the density of pinning centres! Both predictions of equation (38) have been checked 
numerically. The results are plotted in Figure 6: symbols represent the numerical points and the dashed lines, the predicted scalings. The agreement is seen to be correct for both parameters. This confirmation is crucial, since it shows that the proposed scenario for the underlying mechanism of friction (see Sect. 4) is coherent. In particular, this justifies our two conjectures (A) and (B) of Section 4.3.

Now we turn our attention to the other measured quantities. According to equation (11), the stationary friction force $F_{\mathrm{f}}^{\infty}$ is the sum of two term, $\kappa_{\mathrm{L}} / \nu \alpha$ and $\delta F_{0} / \alpha$. However combining the "microscopic" expressions obtained for all the different parameters involved. equations (29, $30,31,36$ ), we obtain that the stationary friction force $F_{\mathrm{f}}^{\infty}$ simply scales like $F_{\text {pin }}$, the r.m.s. value of the fluctuations in equilibrium. Using equation (19), this leads to

$$
\begin{aligned}
F_{\mathrm{f}}^{\infty} & \sim F_{\mathrm{pln}} \sim L \frac{n_{\mathrm{p}}^{2 / 3} f_{0}^{4 / 3}}{k a R_{\mathrm{p}}^{1 / 3}} \\
& \sim n_{\mathrm{p}}^{2 / 3} A_{\mathrm{p}}^{4 / 3} R_{\mathrm{p}}^{-5 / 3}
\end{aligned}
$$

On the other hand, using $(30,31,36)$, the recovery length $\zeta$ is found to scale like

$$
\begin{aligned}
\zeta & =\frac{1}{\nu \alpha} \\
& \sim \frac{F_{\mathrm{p} \text { In }}}{\kappa_{\mathrm{L}}} \sim n_{\mathrm{p}}^{-1 / 3} A_{\mathrm{p}}^{1 / 3} R_{\mathrm{p}}^{-2 / 3}
\end{aligned}
$$

where the microscopic expressions for $F_{\text {pin }}$ and $\kappa_{\mathrm{L}}$ have been used (see Eqs. $(19,32)$ ).

In fact, an equivalent relaxation length can be introduced, defined as the ratio between the asymptotic friction force $F_{\mathrm{f}}^{\infty}$ and the Labusch constant $\kappa_{\mathrm{L}}$ :

$$
\delta \equiv \frac{F_{\mathrm{f}}^{\infty}}{\kappa_{\mathrm{L}}} .
$$

Since $F_{\mathrm{f}}^{\infty}$ scales like $F_{\text {pin }}$, this length exhibits the same scaling as the recovery length $\zeta$ :

$$
\delta \sim \zeta \sim n_{\mathrm{p}}^{-1 / 3} A_{\mathrm{p}}^{1 / 3} R_{\mathrm{p}}^{-2 / 3}
$$

In Figures 7 and 8, we summarize the numerical results for the scalings of the different quantities as a function of the density of pinning centres. The symbols are the numerical points and the dashed lines indicate the predicted scalings. The error bars were estimated in the following way: for a given (random) distribution of pinning centres, the measured quantities were averaged over many forth and back cycles (typically 15), giving a particular value for the desired parameter. Then we averaged again on different random configurations of pinning centres (typically 10): this gives a mean value and a typical error bar for each quantity.

For the pinning force and the Labusch parameter, the error bars are within the size of the symbols and thus not displayed explicitly in the figure. For both parameters, the numerical results (triangles for $\kappa_{\mathrm{L}}$ and crosses for $F_{\mathrm{f}}^{\infty}$ ) are in good agreement with the predicted scalings of equations $(39,34)$ : these are illustrated by the dotted lines in the figure, with slope 1 for $\kappa_{\mathrm{L}}$ and slope $4 / 3$ for $F_{\mathrm{f}}^{\infty}$

Note however the large error bars involved in the estimation of the recovery length, $\zeta$. Indeed, in contrast to the other parameters, the estimated value of $\zeta$ fluctuates a lot when different configurations of pinning centres are considered. Good statistics would therefore involve an average over many different (randomly distributed) configurations of defects. Such a procedure requires much more numerical work and goes beyond the purpose of this first stage 
study, devoted to construct a coherent picture of friction. The numerical results for $\zeta$ (circles) are however coherent with the proposed scaling (40), as shown in Figure 8 . The underlying dotted line has a slope $-1 / 3$, as predicted by equation (40).

On the other hand, the estimate of the other length $\delta$ is obtained to be more precise, since the errorbars involved in the numerical calculations of $F_{f}^{\infty}$ and $\kappa_{L}$ are both quite small. The numerical results (squares) are seen to follow quite closely the predicted scaling of equation (42). illustrated in the figure by a line with a slope $-1 / 3$.

Finally we checked the dependence of the Labusch parameter and of the pinning force as a function of the strength $A_{\mathrm{p}}$ and range $R_{\mathrm{p}}$ of the defects, as given by equations $(32,39)$. The results are summarized in Figures 9 and 10. The dependence of $\kappa_{\mathrm{L}}$ has already been discussed in Section 5.1. The results for the friction force $F_{\mathrm{f}}^{\infty}$ are plotted as triangles in both figures. The predictions of equation (39) are illustrated as dotted lines of slopes $4 / 3$ for the dependence on $A_{\mathrm{p}}$ and $-5 / 3$ for the dependence on $R_{\mathrm{p}}$. Here again, the numerical results are obtained to be in correct agreement with the theoretical predictions. Note that the agreement of the measured $F_{\mathrm{f}}^{\infty}$ and $\kappa_{\mathrm{L}}$ with the predicted scalings on $A_{\mathrm{p}}$ and $R_{\mathrm{p}}$ implies necessarily that $\delta$ follows the predicted dependence on $A_{\mathrm{p}}$ and $R_{\mathrm{p}}$ as displayed on equation (42).

Let us emphasize again that we only performed a partial exploration of the parameter space $\left\{n_{\mathrm{p}}, A_{\mathrm{p}}, R_{\mathrm{p}}\right\}$, and a more complete numerical work is still to be done. However within the explored window, the trend of all the quantities involved in the proposed scenario is obtained to follow the theoretical predictions. Therefore, the numerical results validate with some confidence the proposed picture for the onset of friction.

\section{Discussion and Conclusions}

This work focuses on the onset of friction at the interface between an elastic and a "rough" surface. In particular, we study how the friction force evolves when the system is pulled quasistatically over the surface. We have shown that, in this controlled limit, the system exhibits hysteresis and more surprisingly, history dependent effects. These "memory" effects are characterized by a length scale. While it was known for a while that elasticity is able to produce hysteresis and dissipation, it is shown here that the memory effects originate from the collective character of the induced elastic instabilities.

The collective character of quasistatic friction is related to the existence of a correlation length in the system at equilibrium, which expresses the balance between the elasticity of the medium and pinning to the defects. When an external force is applied to the system, this length is shown to increase linearly with the applied force. This linear increase provides a simple explanation for the exponential approach to the stationary static pinning force. as observed numerically. Moreover the evaluation of this length allowed us to make some predictions for the dependence of the stationary pinning force and recovery length on the microscopic parameters of the system (density, strength and range of the defects of the surface). In particular, it is shown that the value of the out-of equilibrium stationary pinning force is fixed by the typical scale for the fluctuations of the pinning force in equilabrium. The link between the two limits is provided by the analysis of the increase of the out of equilibrium correlation length in the spirit of linear response theory. The predicted scalings are in agreement with the measured numerical results, validating therefore the proposed scenario.

We stress the fact that although our analysis focuses on a one-dimensional problem, the generalization of our results to a two-dimensional system (i.e. a thin layer with a free upper surface) is straightforward. In particular, we expect the proposed scenario leading to the exponential approach of the saturation force to still hold. Only the scalings of the different parameters (as a function of the microscopic characteristics, $n_{\mathrm{p}}, A_{\mathrm{p}}$, . ) will change. The main 
reason for this qualitative equivalence between one and two dimension systems (studied under the present conditions) is that the Larkin correlation length is still finite and smaller than the system size. This can be verified by a simple estimate of the Larkin length. In two dimensions, the latter scales as [26]

$$
\ell_{\mathbf{L}} \sim \frac{C R_{\mathrm{p}}^{2}}{n_{\mathrm{p}}^{1 / 2} A_{\mathrm{p}}}
$$

where $C$ is a combination of the shear $\left(C_{66}\right)$ and compression $\left(C_{11}\right)$ modulus of the $2 \mathrm{~d}$ elastic system. Let us assume that this system is made e.g. of a thin elastic plate of thickness $h$. $C$ is then roughly estimated to be $C \sim E h$, with $E$ the Young's modulus of the elastic media. Just to fix the ideas, we assume moreover that the interaction between the surface and the elastic media takes its origin in the elastic deformation of the asperities of the latter. This leads to $A_{\mathrm{p}} \sim E R_{\mathrm{p}}^{3}$ and then $\ell_{\mathrm{L}} \sim h /\left(n_{\mathrm{p}} R_{\mathrm{p}}^{2}\right)^{1 / 2}$ This rough estimate leads therefore to an equilibrium Larkin length of the order of a few thicknesses $h$. This reasoning may slightly underestimate the correlation length (as is the case of the estimate (18) in our 1d system), but does not lead to Larkin length larger than the system size. This property thus preserves the collective character of the elastic instabilities, which is the crucial point of our study. On the contrary, if a (semi-infinite) three-dimensional body slides over a rough surface, it can be shown that a typical correlation length at the sliding boundary is of the kilometric order of magnitude [29]. Thus on the laboratory (and everyday) length scale, the surface of a threedimensional elastic body can be considered as rigid: only single particle pinning can occur. On the other hand, the collective character is recovered on the earth length scale, as observed in earthquake dynamics [2].

Another interesting point is the apparent qualitative analogy between the history dependent effects described in this paper and the experimental results of reference [7]. In this experiment, the friction force between two surfaces of controlled artificial roughness and elasticity is measured as a function of the relative displacement of the two bodies. The roughness is created by steel spheres (of diameter $2 \mathrm{~mm}$ ) placed on the two surfaces in contact. On both surfaces, the steel spheres are embedded in silicon rubber and can only move horizontally because of the presence of the slider. In the experiments, the upper slider is moved at small constant velocity (of order $1 \mu \mathrm{m} / \mathrm{s}$ ) and the force on the bottom surface is measured by a tranducer. As in our system, the force was measured to circulate through an hysteresis loop and the saturation towards the stationary force was measured to follow an exponential law as a function of the displacement, defining a characteristic recovery length. This qualitative agreement seems indeed attractive but should be handled with care. Indeed in this experiment, the presence of the slider to which the elastic layer is glued certainly screens the elastic interaction and does reduce the collective nature of the instabilities, which was a crucial ingredient of our model. On the other hand, instabilities involving a large number of particles are observed in this system $[7,27]$. This renders the situation quite puzzling and this point should thus be first clarified in order to precise the relevance of our approach to discuss these experimental results.

Our study only focuses on the quasistatic motion of the elastic media, i.e., when any dynamical effect can be neglected. When the system acquires a finite velocity, one may expect that the main lines of the proposed scenario will subsist and could provide an interesting alternative route to dynamical friction. However, such a generalization should be handled with care. In particular, as already emphasized in Section 5.2, our understanding of the creation of instabilities is only qualitative. In the dynamical case and for large sliding velocity, sound waves induced by instabilities may play a crucial role. These effects are not included in our discussion of the quasistatic limit. 
Finally, it is interesting to note that our approach may be generalized to the problem of contact line motion in the quasi-static limit. Fundamentally, these problems are indeed very close: the contact line exhibits (long range) elastic properties due to capillary effects [31] and is able to pin to the impurities of the solid surface (like e.g. the chemical heterogeneities of the surface or the geometrical roughness) (see [32] for details). Note that although the triple line is $1 \mathrm{D}$, the elastic distortion is in fact 2D (like the liquid-gaz interface) [31], but reduces to a $1 \mathrm{D}$ problem in a confined geometry (i.e. in the so-called Hele-Shaw cell) [33]. Experimentally, the quasistatic motion of a contact line has been investigated very recently on nanoscales, using the Surface Force Apparatus (SFA) technique [10]. The experimental results present a striking analogy with those obtained in the experiments of reference [7] on quasistatic friction between artificial surfaces (discussed in the previous paragraph). In particular, the force acting on the contact line (related to the contact angle of the line) is shown to present hysteresis as a function of the displacement of the contact line, defining spatial memory effects characterized by a length scale. Moreover, the approach to the stationary value of the force (i.e., of the advancing or receding contact angle) was obtained experimentally to be exponential too. Obviously, all these facts are very similar to the features described in the present paper. Beyond this encouraging agreement, further work is needed to understand more deeply these analogies and characterize more precisely the regime in which these analogies are relevant. We are currently studying these problems.

More generally, it is interesting to note that a detailed description of instabilities (here elastic instabilities) is not needed to understand qualitatively the onset of friction. The hope would then be to be able to include plastic effects into the scenario too, along the same lines as elastic effects. Work along these lines is in progress.

\section{Acknowledgments}

The authors would like to thank Jérôme Crassous and Elisabeth Charlaix for communicating their experimental results prior to publication. L.B. is grateful to Joergen Vitting Andersen and Christiane Caroli for helpful discussions. L.B. acknowledges support of a NATO grant for scientific research during his stay at Imperial College, London.

\section{References}

[1] Bowden F. and Tabor D., Friction and Lubrication of solids (Clarendon Press, Oxford, 1950).

[2] Scholz C.H., The Mechanics of Earthquakes and Faulting (Cambridge University, Cambridge, 1990).

[3] Rice J.L. and Ruina A.L., J. Appl. Mech. 50 (1983) 343.

[4] Heslot F., Baumberger T., Caroli B. and Caroli C., Phys. Rev. E 49 (1994) 4973.

[5] Persson B.N.J. and Tossati E., Physics of sliding surfaces, vol. 311 of NATO ASI, B.N.J. Persson and E. Tosatti, Eds. (Kluwer Academic publishers, Dordrecht, 1996).

[6] Caroli C. and Nozieres P., pp. 27-50 in reference [5].

[7] Crassous J., Charlaix E., Ciliberto S. and Laroche C., proceedings of HLRZ Workshop on Friction, Arching and Contact dynamics, Jülich, October 1996, Grassberger, Ed. (World Scientific, Singapore, 1996). 
[8] Scherer V. and Arnold W., Proceedings of the NATO ASI Conference on Micro- and Nanotribology and its Application, Sesimbra, Portugal (1996).

[9] Göddenhenrich T., Müller S. and Heiden C., Rev. Scı. Instr. 65 (1994) 2870.

[10] Crassous J., Loubet J.-L. and Charlaix E., Phys. Rev. Lett. 78 (1997) 2425.

[11] Rabinowicz E., Proc. Phys. Soc. London 71 (1958) 668

[12] Dieterich J.H., J. Geophys. Res. 84 (1979) 2161.

[13] Ruina A.L., J. Geophys. Res. 88 (1983) 10359.

[14] Tanguy A. and Roux S., Phys. Rev. E 55 (1997) 2166.

[15] Tomlinson G.A., Philos. Mag. 7 (1929) 905.

[16] Sokoloff J.B., Surf. Sci. 144 (1984) 267.

[17] Jensen H.J., Brass A. and Berlinsky A.J., Phys. Rev. Lett. 60 (1988) 1676; Brass A., Jensen H.J., and Berlinsky A.J., Phys. Rev. B 39 (1989) 102; Jensen H.J., Brechet Y. and Doucot B., J. Phys. I France 3 (1993) 611.

[18] Cule D. and Hwa T., Phys. Rev. Lett. 77 (1996) 278.

[19] Narayan O. and Fisher D., Phys. Rev. B 48 (1993) 7030.

[20] Leschhorn H., Nattermann T., Stepanow S. and Tang L.-H., Ann. Physik 6 (1997) 1 and references therein.

[21] see Section 2.1. of Jensen H.J., J. Phys. A: Math. Gen. 28 (1995) 1861.

[22] R. Labusch, Cryst. Lattice Defects 1 (1969) 1.

[23] Jensen H.J., Brechet Y. and Brass A., J. Low Temp. Phys. 74 (1989) 293.

[24] Pla O. and Nori F., Phys. Rev. Lett. 67 (1991) 919.

[25] Larkin A.I. and Ovchinnikov Y.N., J. Low. Temp. Phys. 34 (1979) 409.

[26] Tinkham, Introduction to superconductivity (Mc Graw-Hill, Inc., 2nd edition, 1996) pp. 348-353.

[27] Ciliberto S. and Laroche C., J. Phys. I France 4 (1994) 223.

[28] Di Meglio J.-M., Europhys. Lett. 17 (1992) 607.

[29] Persson B.N.J. and Tosatti E., pp. 179-190 in reference [5]

[30] Love A.E., A Treatise on the Mathematical Theory of Elasticity (Dover Publication, New York, 1927).

[31] Joanny J.F. and de Gennes P.G., J. Chem. Phys 81 (1984) 552.

[32] Leger L. and Joanny J.F., Rep. Prog. Phys. 55 (1992) 431.

[33] Limat L., C. R. Acad. Scı. Parzs 413 (1992) 1011. 